Service social

\title{
Le développement des communautés et la santé publique au Québec
}

\section{Denis Bourque et Louis Favreau}

Volume 50, numéro 1, 2003

URI : https://id.erudit.org/iderudit/011352ar

DOI : https://doi.org/10.7202/011352ar

Aller au sommaire du numéro

Éditeur(s)

École de service social de l'Université Laval

ISSN

1708-1734 (numérique)

Découvrir la revue

Citer cet article

Bourque, D. \& Favreau, L. (2003). Le développement des communautés et la santé publique au Québec. Service social, 50(1), 295-308.

https://doi.org/10.7202/011352ar
Résumé de l'article

Le développement des communautés connaît une notoriété nouvelle depuis qu'il est devenu une stratégie du Programme national de santé publique du Québec en 2003. Cet article cherche à clarifier un certain nombre de notions associées au concept de développement des communautés, quelques conditions qui favorisent les pratiques dans ce domaine ainsi que certains enjeux qui y sont liés. Le défi est de mettre les ressources du réseau de la santé au service du développement démocratique des communautés plutôt que d'utiliser les communautés pour atteindre des objectifs de santé publique. À cette fin, une approche est proposée qui consiste à additionner les expertises (expertise de processus, de contenu et expertise citoyenne) et à composer avec des logiques paradoxales afin que le développement des communautés s'inscrive dans une véritable perspective d'organisation communautaire et puisse jouer un véritable rôle dans l'amélioration de la santé et du bien-être des collectivités. 


\section{Le développement des communautés et la santé publique au Québec}

DENIS BOURQUE

Professeur

Département de travail social et des sciences sociales Université du Québec en Outaouais

Courriel : denis.bourque@uqo.ca

LOUIS FAVREAU

Professeur titulaire Chaire de recherche du Canada en développement des collectivités de l'Université du Québec en Outaouais

Courriel : louis.favreau@uqo.ca

Le développement des communautés connaît une notoriété nouvelle depuis qu'il est devenu une stratégie du Programme national de santé publique du Québec en 2003. Cet article cherche à clarifier un certain nombre de notions associées au concept de développement des communautés, quelques conditions qui favorisent les pratiques dans ce domaine ainsi que certains enjeux qui y sont liés. Le défi est de mettre les ressources du réseau de la santé au service du développement démocratique des communautés plutôt que d'utiliser les communautés pour atteindre des objectifs de santé publique. À cette fin, une approche est proposée qui consiste à additionner les expertises (expertise de processus, de contenu et expertise citoyenne) et à composer avec des logiques paradoxales afin que le développement des communautés s'inscrive dans une véritable perspective d'organisation communautaire et puisse jouer un véritable rôle dans l'amélioration de la santé et du bien-être des collectivités.

Mots clés : développement, communautés, santé publique, organisation communautaire, développement social, empowerment, CLSC.

Since becoming one of the strategies put forth in Quebec's National Public Health Programme, community development has gained a higher profile. This article seeks to clarify a certain number of concepts, a few of the conditions that favour the practices of community development, as well as certain issues at stake in it. The challenge is to make the health system's resources serve democratic community development, rather than to have communities used to attain public health goals. To this end, the different forms of expertise (in regard to process, content, and citizenship) must be brought together, otherwise community development will not fully play its role in improving the health and welfare of communities.

Key words: development, communities, empowerment, LCSC (CLSC), public health, community organizing, social development. 
La santé publique au Québec exerce un ascendant grandissant sur l'intervention sociale, que ce soit sous le mode de l'intervention personnelle au moyen des programmes d'intervention précoce (Parazelli et al., 2003) ou sous le mode de l'intervention communautaire (Lachapelle, 2003) au moyen de la stratégie dite du développement des communautés. Or, les pratiques de développement social et économique des collectivités à l'échelon local et régional sont vivantes au Québec depuis longtemps, particulièrement depuis les années 1980 en réponse aux contrecoups de la crise économique structurelle et de la globalisation montante. Depuis peu, ces pratiques font l'objet d'une reconnaissance nouvelle en devenant officiellement une stratégie de santé publique dans le Programme national de santé publique 2003-2012 du ministère de la Santé et des Services sociaux (2003). La reconnaissance du développement des communautés comme stratégie de santé publique s'inscrit dans la continuité des principes d'engagement auprès des communautés et d'action sur les déterminants de la santé présents dans la Politique de la santé et du bien-être (1992) et à l'intérieur des Priorités nationales de santé publique 1997-2002 (1997). Déjà, la Charte d'Ottawa de 1986 (Ottawa, 1986) avait contribué à créer des conditions favorables à la mise en œuvre des pratiques communautaires en santé publique. On comprend de plus en plus que la prévention et la promotion de la santé ne peuvent s'appuyer sur le seul renforcement du potentiel des personnes et qu'il faut aussi adopter une approche de développement des communautés (Fréchette, 2001).

Malgré les nombreux travaux qui ont été publiés dans les dernières années sur le sujet (Bélanger et al., 2000; CSBE, 2001; Lachapelle, 2001, 2003; INSP, 2002a,b,c,d; Favreau et Fréchette, 2003b), le concept de développement des communautés exige encore des clarifications. Ainsi, le Programme national de santé publique approfondit peu la question, n'en propose aucune définition et annonce un document complémentaire qui devait présenter en détail la stratégie relative au développement des communautés (MSSS, 2003, p. 22), mais qui n'est toujours pas publié dix-huit mois plus tard. Pourtant, les CLSC sont tenus par la loi (Québec, 2001) de mettre en œuvre, d'évaluer et de mettre à jour régulièrement, de concert avec les organismes communautaires, un plan d'action local de santé publique dont le développement des communautés constitue l'une des stratégies de base et, dans certaines régions, un domaine spécifique d'intervention ${ }^{1}$. Les CLSC et leurs organisateurs communautaires doivent composer avec le flou entourant la conceptualisation et l'opérationnalisation du développement des communautés dans le Programme national de santé publique. Voilà qui nous invite à tenter de clarifier, dans la première partie de cet article, un certain nombre de notions associées au concept de développement des communautés, particulièrement sur le plan de l'orientation et des valeurs qu'elles supposent. Par la suite, nous chercherons à cerner quelques conditions opérationnelles qui favorisent des pratiques de développement des communautés

1. Les plans d'action régionale en santé publique, qui relèvent des agences régionales de développement des réseaux locaux de santé et de services sociaux, ont opté pour inclure le développement des communautés soit comme stratégie dans chacun des six domaines d'intervention en santé publique (santé environnementale, adaptation et intégration sociale, habitudes de vie, etc.), soit comme un septième et distinct domaine d'intervention. 
susceptibles de s'inscrire dans une perspective d'organisation communautaire. Nous examinerons tout d'abord des conditions stratégiques, puis des conditions pratiques, dont les enjeux concernent l'amélioration de la santé et du bien-être des collectivités.

\section{Le développement des communautés : un concept porteur de sens et de valeurs}

Le concept de développement des communautés trouve son origine aux États-Unis, où le community development (Christenson et al., 1989) fut utilisé par les pouvoirs publics locaux et par les mouvements associatifs comme stratégie économique de lutte contre la pauvreté, en particulier par la revitalisation des centres urbains des cités nord-américaines. Le développement des communautés a aussi constitué une stratégie de participation des populations de pays en développement à certains programmes de développement social et économique mis en œuvre par des organisations internationales après la Deuxième Guerre mondiale (Doucet et Favreau, 1991). Au Québec, le développement des communautés tire ses origines de l'organisation communautaire, particulièrement de son modèle de développement local de type communautaire qui se caractérise par la transformation des problèmes collectifs en projets collectifs en misant sur la mobilisation des communautés locales, en partenariat avec les principaux acteurs des différents secteurs (santé et services sociaux, éducation, emploi, économie, municipalités, etc.).

Le concept de développement des communautés renvoie à deux notions de base : développement et communauté. Pour Favreau et Fréchette (2002, p. 31) : «Le développement est aujourd'hui [...] conçu comme une mobilisation économique, sociale et culturelle de toutes les potentialités d'un pays (ou d'une région, ou d'une communauté locale) autour d'un certain nombre d'objectifs d'amélioration des conditions de vie des populations. » Cette définition rejoint celle du Conseil de la santé et du bienêtre (CSBE, 2001, p. 11) selon lequel «le développement est un processus, une démarche par laquelle une communauté, par voie d'initiatives, tente de maintenir ou d'améliorer, selon les valeurs qu'elle juge prioritaires, les conditions de vie collectives et/ou individuelles ». Le développement présente donc un caractère fortement endogène, puisqu'il se base sur des initiatives et des valeurs issues du milieu et qu'il cible les conditions de vie de ce milieu. Le sens du développement provient donc fondamentalement des communautés et des acteurs qui les composent. On peut synthétiser la pensée actuelle sur le développement en retenant, parmi d'autres, les variables suivantes (Bartoli, 1999, p. 1): le social doit être au poste de commande; 2) l'économie doit être considérée pour ce qu'elle est, un instrument de développement et non une fin; 3) l'environnement doit constituer une condition nouvelle et incontournable dans les choix économiques qui s'opèrent; 4) la poursuite simultanée de quelques grands axes d'intervention s'imposent, notamment l'emploi, le logement, l'inclusion sociale, la santé, etc.

Quant à la notion de communauté, elle renvoie à un «regroupement de personnes vivant sur un territoire donné et partageant des intérêts communs à l'échelle de ce territoire »(CSBE, 2001, p. 11). Même si les individus peuvent appartenir à la fois à 
des communautés d'intérêts, d'identités et de territoires, c'est la dimension territoriale qui est privilégiée dans la perspective du développement des communautés (tout en n'excluant pas les autres types de communautés). L'ancrage territorial des communautés joue en effet un rôle central sur le plan du développement, car il constitue un moteur de l'action collective. II permet aussi d'aborder la réalité d'une façon globale (dans ses dimensions sociales, économiques, environnementales, culturelles) en ciblant la communauté dans son ensemble, plutôt qu'une somme de groupes vulnérables, ce qui favorise le décloisonnement des interventions et augmente leur impact par leur portée plus transversale. Selon White (1994, p. 44), le concept de communauté peut être vu sous deux angles : la communauté existentielle, comprise comme un espace social où des groupes peuvent se constituer et exprimer leur identité, et la communauté instrumentale "définie en fonction de ce qu'elle fait plutôt que de sa signification pour ses membres ». La conception de la communauté en santé publique pourrait inclure ces deux types de communautés qui, selon White (1994, p. 44), ne sont pas mutuellement exclusifs, mais renvoient à «des espaces sociaux façonnés simultanément par des frontières symboliques et par des frontières bureaucratiques ». Cependant, mettre l'accent uniquement sur la conception instrumentale de la communauté aurait des conséquences significatives au plan de l'organisation communautaire, que nous examinerons plus loin.

Lorsque l'on regroupe les notions de développement et de communauté, on rejoint le concept de développement des communautés défini ainsi par l'Institut national de santé publique (INSPQ) : "le développement des communautés est en fait un processus de coopération volontaire, d'entraide et de construction de liens sociaux entre les résidents et les institutions d'un milieu local, visant l'amélioration des conditions de vie sur les plans physique, social et économique " (INSPQ, 2002a, p. 16) ${ }^{2}$. Le développement des communautés est donc pluridimensionnel et se veut un concept intégrateur sur le plan local des notions de développement social, de développement économique, de développement culturel et environnemental qui conduit au développement humain durable (Bégin, 2002). Le développement social se déploie au niveau de la société, des régions et des communautés en reposant sur les notions de participation sociale et de démocratisation au moyen de structures politiques et de programmes sociaux qui permettront aux citoyens de participer activement à la vie sociale et d'exercer entièrement leur citoyenneté au sein d'une société démocratique (CSBE, 1997; INSPQ, 2002d). Quant au développement des communautés, il implique également la participation sociale, mais en ciblant la capacité d'une communauté locale d'agir sur sa réalité et de prendre en charge son développement avec comme conséquence positive d'améliorer sa santé et son bien-être. En fait, le développement des communautés est un indicateur de santé en lui-même, car la santé est le corollaire de la mobilisation active des communautés. Citons à ce chapitre l'exemple de cette collectivité, frappée par la fermeture du principal employeur de la ville, qui décide de mettre sur pied une clinique communautaire de santé pour préve-

2. Cette définition qui date de 2002 nous semble très pertinente et aurait pu être reprise par le Programme national de santé publique de 2003. Or, au lieu de cela, le Programme national reste muet sur la définition du développement des communautés. 
nir les problèmes de santé liés au chômage massif (stress, maladies cardiaques, dépressions). Des chercheurs ont évalué que la mobilisation communautaire associée au projet avait eu un impact positif plus significatif sur la santé de la population que les services fournis à la clinique (Bélanger et al., 2000, p. 37).

Des initiatives de développement des communautés naissent partout au Québec dans des domaines aussi variés que la sécurité alimentaire, l'emploi, le logement, le décrochage scolaire, le transport, l'amélioration de la qualité de l'air, de l'eau, etc. Citons, à titre d'exemple, les corporations de développement économique communautaire (CDEC), les maisons de quartier ${ }^{3}$, les corporations de développement communautaire (CDC), les tables de concertation sur la faim, celles sur le développement social, les comités Villes et Villages en santé, etc.

\section{Intervention en développement des communautés}

Le développement des communautés peut certes se réaliser sans la contribution des directions de santé publique et des CLSC, mais avec des ressources plus limitées. II y a donc développement des communautés, d'une part, et intervention en développement des communautés, d'autre part. On reconnaît généralement que l'intervention en développement des communautés comporte un certain nombre de caractéristiques incontournables (INSPQ, 2002a). Nous en retenons deux :

1. La participation des populations et des acteurs locaux au développement des communautés, qui implique un développement par et avec les communautés et non pour les communautés. La participation vise l'appropriation du développement par les communautés, telle qu'elle est définie par le CSBE (2001, p. 11) pour qui «l'appropriation consiste, pour une communauté, à pouvoir régler les problèmes qu'elle estime les plus importants en appliquant les solutions qu'elle juge les mieux adaptées ». L'intervention en développement des communautés se caractérise donc par l'empowerment ${ }^{4}$ communautaire qui vise à rendre les communautés acteurs et sujets de leur développement (et non objet) en les habilitant à faire des choix, à prendre des décisions et à passer à l'action dans le but d'exercer un meilleur contrôle sur leur devenir.

2. Cette intervention se caractérise aussi par la concertation et le partenariat rendus nécessaires par la complexité des problèmes auxquels sont confrontées les communautés locales. Le développement des communautés s'inscrit dans une vision globale qui cherche à dépasser les barrières érigées entre les secteurs, particulièrement entre l'économique et le social, en misant sur l'addition des forces présentes dans les communautés. La réussite du développement des communautés repose en bonne partie sur leur capacité à assurer la concertation des acteurs concernés et à traduire

3. L'expérience d'intervention de quartier dans Jean-Dallaire à Hull est particulièrement éloquente à cet effet, comme le montrent Favreau et Fréchette (2003a).

4. L'empowerment désigne une capacité d'agir concrètement et de façon autonome ainsi que le processus pour atteindre cette capacité d'agir (Ninacs, 1995). 
cette concertation en partenariat, c'est-à-dire en projets et actions librement convenus afin de répondre aux besoins reconnus conjointement.

Ces caractéristiques de l'intervention sont compatibles avec le rôle que le Programme national de santé publique (PNSP) attribue à la santé publique et aux acteurs publics en ce qui concerne le développement des communautés, soit :

[...] de favoriser et de soutenir la participation des personnes qui composent ces communautés à un processus visant à déterminer les problèmes de santé les plus importants pour elles et les solutions les plus appropriées à leur apporter; il s'agit en fait de soutenir le processus d'empowerment des collectivités. Cette stratégie suppose l'engagement des acteurs locaux et régionaux, le recours à l'approche communautaire et le partenariat avec les autres secteurs en vue de favoriser la réalisation de projets issus des communautés et axés sur leur santé et leur bien-être (MSSS, 2003, p. 22).

En somme, comme le fait remarquer l'Institut national de santé publique, le concept de développement des communautés ne véhicule pas une vision unique et restrictive des concepts et «a le mérite d'englober tous ces concepts dans la mesure où on l'utilise pour désigner l'ensemble des approches d'action communautaire fondées sur la participation des membres d'une communauté et sur le développement de leur emprise collective sur leurs ressources et leurs institutions locales » (INSPQ, 2002b, p. 26). II est donc possible de retenir une formule simple pour définir le développement des communautés : toutes formes d'action communautaire structurée dans un territoire local qui, par la mobilisation démocratique des citoyens et des acteurs sociaux, ciblent des problèmes collectifs et dont les résultats auront un impact positif sur les conditions et la qualité de vie. Le développement des communautés comporte donc deux dimensions : sa dimension de processus, qui consiste à mettre en marche et à soutenir l'action communautaire, et sa dimension de résultats, c'est-à-dire la mise en place de réponses collectives aux priorités d'un milieu sous forme de ressources, de services, de dispositifs, etc. La dimension la plus déterminante demeure celle du processus, car sans l'appropriation démocratique par les communautés des enjeux locaux de santé les résultats risquent fort d'être inexistants ou inappropriés.

Toutefois l'intervention en développement des communautés requiert un certain nombre de conditions opérationnelles dont nous soulignerons les plus déterminantes à notre point de vue.

\section{L'addition des expertises : une condition stratégique}

Tout d'abord, il importe de reconnaître l'expertise spécifique des CLSC en développement des communautés, puisque ceux-ci représentent l'un des plus importants acteurs institutionnels de développement des communautés au Québec des trente dernières années (Favreau et Hurtubise, 1993). Ces établissements et leurs organisateurs communautaires sont à l'origine ou contribuent à des réussites telles que le développement d'une grande partie du mouvement communautaire autonome, les 
pratiques de développement économique communautaire, les dispositifs d'insertion socioprofessionnelle comme les carrefours jeunesse-emploi (Assogba, 2000), les différentes instances locales de concertation, la lutte à la pauvreté (Fréchette, 2000), etc. Les CLSC et leurs organisateurs communautaires ont développé une expertise liée au processus de développement des communautés. Les CLSC sont de plus l'un des acteurs locaux engagés en tant qu'institution dans ce développement. D'ailleurs, le Conseil de la santé et du bien-être (CSBE) recommande que «le rôle des CLSC pour soutenir localement et en partenariat le développement des communautés soit reconnu et intensifié comme action efficace à l'amélioration de la santé et du bienêtre et à la prévention des problèmes sociaux et de santé des personnes et des communautés, et que des ressources y soient protégées à cette fin » (CSBE, 2001, p. 40).

La seconde expertise à considérer est celle des directions et des professionnels de santé publique. II s'agit d'une expertise de contenu par rapport à l'expertise de processus des CLSC (Hétu, 2003). Cette expertise concerne la connaissance de l'état de santé des populations, la définition des grands objectifs d'amélioration de la santé sur les plans national et régional, la référence aux programmes et modèles d'intervention jugés scientifiquement efficaces, l'évaluation des projets de développement des communautés sur le plan des processus et des résultats, le soutien technique et financier aux nouveaux projets de développement et à ceux existants, etc.

Une troisième expertise, qui est celle des communautés elles-mêmes, concerne la pertinence même des projets de développement. II s'agit d'une expertise citoyenne qui passe particulièrement par l'action communautaire autonome et qui s'exerce dans la définition des problèmes ainsi que dans le choix des priorités et des moyens d'action. Si l'expertise de contenu relève de la science, l'expertise citoyenne relève des valeurs et de la conscience (Thibault et al., 2000). L'expertise de contenu peut éclairer, mais elle ne décide pas des valeurs et des orientations des communautés. Refuser de reconnaître cette expertise citoyenne équivaut à instaurer un rapport de pouvoir au détriment des communautés, rapport basé sur la position épistémologique voulant qu'il y ait «ceux qui savent » définir les problèmes, les besoins et les programmes d'intervention et «ceux qui ne savent pas » (Parazelli et al., 2003, p. 89). La reconnaissance de l'expertise citoyenne devient une condition de l'appropriation par les communautés de leur développement. Mais cette appropriation peut se renforcer de la contribution adéquate des expertises de processus et de contenu. Le Conseil de la santé et du bien-être (CSBE, 2001, p. 57) reconnaît d'ailleurs comme un facteur favorable aux projets de développement le fait de pouvoir recourir à une expertise extérieure.

\section{Composer avec des logiques paradoxales}

Une seconde condition d'opérationnalisation stratégique du développement des communautés consiste pour les CLSC à naviguer au cœur d'une dynamique complexe que l'on peut illustrer ainsi : 
Logique descendante

Logique d'expertise prescriptive

Logique d'empowerment

CLSC/Organisation communautaire

Logique autonomiste

Logique complémentariste

Logique ascendante

Dans ce schéma, inspiré de Duperré (1992), on retrouve la logique descendante qui correspond aux politiques et programmes gouvernementaux de recours aux communautés et qui se subdivise en deux logiques : la logique d'expertise prescriptive, qui s'appuie sur le pouvoir qu'ont les experts de déterminer le contenu des programmes pour répondre aux besoins prédéterminés des communautés, et la logique d'empowerment, qui renvoie à la mobilisation active des communautés pour agir sur les déterminants de la santé. Ces deux logiques sont particulièrement présentes dans le domaine de la santé publique.

À l'opposé, on retrouve à la base du schéma la logique ascendante, qui correspond à l'aspiration des acteurs locaux de pouvoir déterminer localement les problèmes prioritaires et les stratégies d'action appropriées. Cette logique ascendante se subdivise elle-même en deux logiques (Proulx, 1997) : la logique autonomiste, qui est faite d'exigences d'autodétermination des pratiques, et la logique complémentariste, qui renvoie à la demande d'institutionnalisation et d'intégration aux programmes issue de la logique descendante, souvent à des fins de survie financière (Fournier et al., 2001).

Au centre, se retrouvent les CLSC (et leurs organisateurs communautaires), en interface entre les logiques descendante et ascendante, ainsi qu'en situation de double imputabilité : vers le bas et leur milieu ainsi que vers le haut et l'appareil de planification régionale et nationale. En effet, d'une part, les CLSC sont parties prenantes des politiques et programmes descendants; ils doivent, de ce fait, tenir compte des exigences du Programme national de santé publique (PNSP) et du Plan d'action régional (PAR) de santé publique. D'autre part, les CLSC sont des experts du processus et des acteurs locaux du développement des communautés. A ce titre, ils doivent assurer l'adaptation des programmes et des plans descendants aux réalités locales (particulièrement en termes de prise en compte des dynamismes de concertation, du rythme de travail, etc.). Les CLSC ont donc un rôle majeur à jouer pour que le développement des communautés fasse l'objet d'une régulation horizontale plutôt que d'une régulation verticale, considérée comme un facteur défavorable à ce développement (CSBE, 2001, p. 58). Le développement des communautés peut, en effet, 
difficilement se prescrire ou se décréter de l'extérieur, ou être le résultat de programmes de planning social ou de marketing social, parce que ces modèles ne misent ni sur la participation, ni sur la concertation ou sur l'empowerment des communautés.

À première vue, on pourrait conclure à l'incompatibilité entre une approche «topdown » comme peut l'être le Programme national de santé publique (et les programmes d'action régionale en santé publique) et une approche «bottom-up», comme l'exige le développement des communautés. II s'agit précisément du défi des CLSC que de se situer de manière efficace à la jonction entre les logiques descendantes et ascendantes, en assumant un rôle de leadership démocratique (donc ni passif ni directif). Cette contribution stratégique des CLSC fera et fait déjà en sorte que, sur le terrain, le développement des communautés est un processus négogène ${ }^{5}$, c'est-àdire en partie exogène, parce qu'il est influencé par les programmes, par l'expertise de contenu et par le soutien technique et financier en provenance des appareils de planification, et en partie endogène, parce qu'il repose sur la mobilisation et sur l'appropriation des communautés. Un processus strictement exogène renverrait à un développement directif des communautés peu efficace, puisque de nombreuses recherches révèlent que la participation des populations concernées donne lieu à de meilleures interventions et à de meilleurs résultats en matière de santé (Bélanger et al., 2000). Par ailleurs, un processus strictement endogène serait également fragilisé du point de vue de son efficacité, puisque privé alors des leviers, des ressources et de l'expertise externes. Dans tous les programmes de santé publique et dans tous les plans d'action locaux de santé publique (PAL), le processus négogène pourrait offrir les meilleures chances de succès dès que l'on fait appel au développement des communautés.

\section{Des conditions pratiques au développement des communautés}

Le développement des communautés est un outil puissant d'amélioration de la santé des personnes et des collectivités (Bélanger et al., 2000), mais c'est aussi un processus fragile et délicat qui peut facilement être enrayé même avec les meilleures intentions du monde. Ce processus exige soutien, ressources et expertises qui doivent résolument se mettre au service du processus plutôt que de chercher à le déterminer dans l'une ou l'autre de ses composantes ou de ses étapes. Le Conseil de la santé et du bien-être fait un constat similaire à propos de l'appropriation par les communautés de leur développement :

Nous avons constaté que les facteurs favorables à l'appropriation sont le plus souvent à l'intérieur des communautés et que les facteurs défavo rables se situent le plus souvent à l'extérieur de celles-ci.[...] Les difficultés d'appropriation des communautés sont liées, d'une part, à leurs propres limites de participation et à leur vulnérabilité et, d'autre part, à l'intervention inappropriée de l'État (CSBE, 2001, p. 8, 39).

5. L'expression est de Claudine Papin de la Coopérative Tandem et a été entendue lors de la Rencontre régionale du développement local tenue à Gatineau le 6 juin 2003. 
Le Programme national de santé publique (MSSS, 2003, p. 19) définit des repères pour une éthique en santé publique au nombre desquels figure le principe « de nonmalfaisance qui sous -tend l'obligation morale de ne pas nuire ni causer du tort aux autres ; il rappelle l'importance de protéger la population contre les méfaits, iatrogéniques ${ }^{6}$ ou autres, découlant de certaines actions ou de l'omission d'agir ». Au nom de ce principe, il serait important d'éviter de recourir à des approches inappropriées en matière de développement des communautés qui pourraient provoquer, à la limite, de la démobilisation. Aussi vaut-il mieux une initiative ou un projet qui mobilise les acteurs locaux plutôt qu'un programme évalué scientifiquement, mais auquel la communauté ne s'identifie pas ou qui ne correspond pas aux particularités du milieu.

Par ailleurs, le développement des communautés nécessite souvent des ressources humaines compétentes soit pour en prendre l'initiative, l'animer ou le soutenir, car " la mobilisation de la communauté et l'autodétermination nécessitent un soutien et un accompagnement fréquents » (INSPQ, 2002b, p. 17). De plus, il faut pouvoir compter sur des ressources financières et matérielles pour permettre la réalisation concrète des projets et des initiatives issus des milieux. Or, le Programme national de santé publique et les plans d'action régionaux de santé publique ne sont pas assortis du financement requis en ce qui concerne le développement des communautés. Pourtant, la commission Clair avait reconnu la responsabilité publique en la matière :

\begin{abstract}
Le lien entre la santé et les conditions de vie n'est plus à démontrer. Nous savons aujourd'hui qu'il est possible, voire nécessaire, d'agir collectivement sur les grands déterminants de la santé, notamment sur les habitudes de vie, l'éducation, l'emploi, le revenu et l'environnement. C'est à l'État qu'il revient d'amorcer les activités de prévention, de protection et de promotion de la santé et de mettre en place les conditions susceptibles de développer la capacité des individus et des collectivités à se prendre en charge (Québec, 2000, p. 7).
\end{abstract}

Le développement des communautés ne peut servir de paravent à l'obligation d'agir au plan national sur les déterminants socioéconomiques de la santé. L'action intersectorielle ne peut être une approche valable seulement au plan local, là où les moyens et l'influence sur les politiques publiques sont les plus faibles. Lachapelle (2004, p. 10) soulève le risque d'un désengagement des obligations de la santé publique qui s'en remettrait à un développement des communautés sans moyens, alors que «les actions locales ont besoin de politiques nationales et de support au niveau régional ". L'insuffisance des moyens ne devrait toutefois pas empêcher de saisir l'opportunité que peut représenter le développement des communautés dans la perspective de la santé publique en termes de reconnaissance et de développement des pratiques d'action et d'organisation communautaires. II serait donc possible de capitaliser sur l'intervention de développement des communautés en santé publique pour intensifier des interventions existantes ou pour renouveler des pratiques. L'initiative appartient aux CLSC qui ont tout intérêt à le faire en lien étroit avec le

6. latrogénique signifie provoqué par le médecin [Note des auteurs]. 
mouvement communautaire autonome ainsi qu'avec les structures existantes de mobilisation ou de concertation locale et intersectorielle.

\section{Conclusion}

Les pratiques de développement des communautés seront désormais influencées au Québec par les programmes et le financement en provenance de la santé publique. Or, le sens du développement des communautés ne peut venir ultimement que des communautés et des acteurs qui les composent. Comme le souligne le Regroupement québécois des intervenants et intervenantes en action communautaire en CLSC et en centre de santé (RQIIAC), le défi de l'intervention en développement des communautés « est de mettre le réseau de la santé au service des communautés plutôt que de simplement mettre les communautés à contribution pour atteindre des objectifs de santé publique » (Lachapelle, 2003, p. 28). Il y a nécessité de le faire en tenant compte de l'approche de haut en bas que semblent adopter certaines directions régionales de santé publique. Lachapelle (2004, p. 9) soulève l'exemple d'un plan d'action régional en santé publique qui part des experts pour rejoindre la population. II ajoute que cette direction de santé publique « donne le message qu'elle sait bien ce qui convient aux communautés locales et précise les actions que les projets locaux doivent concrétiser ». Pour renverser cette tendance, il faudra arriver à additionner les expertises et à composer avec les logiques paradoxales, à défaut de quoi le développement des communautés ne pourra jouer son rôle dans l'amélioration de la santé et du bien-être des collectivités.

\section{RÉFÉRENCES BIBLIOGRAPHIQUES}

Assogba, Y. (2000). Insertion des jeunes, organisation communautaire et société. L'expérience fondatrice des CJE, Sainte-Foy, Presses de l'Université du Québec.

BARTOLI, H. (1999). Repenser le développement, en finir avec la pauvreté, Paris, UNESCO/ Économica, $206 \mathrm{p}$.

BÉGIN, C. (2002). Une toile à tisser... pour favoriser la qualité de vie de la population et le développement local : perspective pour orienter l'action!, Service de la prévention et de la promotion, Régie régionale de la santé et des services sociaux de Lanaudière, $9 \mathrm{p}$.

BÉLANGER, J.-P., R. SulLIVAN et B. SÉVIGNY (2000). Capital social, développement communautaire et santé publique, Montréal, Édition ASPQ, 223 p.

BOURQUE, D. (2003). Essai de typologie du partenariat dans le secteur de la santé et des services sociaux au Québec, Cahier du Centre d'étude et de recherche en intervention sociale (CÉRIS), série Recherches, $n^{\circ} 24$, Université du Québec en Outaouais. 
BouRque, D., et L. FAVREAU (2003). Développement des communautés, santé publique et CLSC, Cahier du Centre d'étude et de recherche en intervention sociale (CÉRIS), série Conférences, $n^{\circ}$ 8, Université du Québec en Outaouais, 23 p.

Christenson, J., et J.W. RoBinson (1989). Community Development in Perspective, Ames, lowa State University Press, 398 p.

CONSEIL DE LA SANTÉ E DU BIEN-ÊTRE (1997). Forum sur le développement social : présentation de la démarche, Québec, CSBE, 12 p.

CONSEIL DE LA SANTÉ E DU BIEN-ÊTRE (2001). L'appropriation par les communautés de leur développement, Québec, CSBE, 52 p.

DOUCET, L., et L. FAVREAU (1991). Théorie et pratiques en organisation communautaire, Sainte-Foy, Presses de l'Université du Québec, 486 p.

DUPERRÉ, M. (1992). «Du discours à la réalité dans le partenariat public-communautaire en santé mentale: une expérience au Saguenay-Lac-Saint-Jean », Nouvelles pratiques sociales, vol. 5, n² 2, Sainte-Foy, Presses de l'Université du Québec, p. 131-146.

FAVReAu, L., et L. FrÉCheTte (2002). Mondialisation, économie sociale, développement local et solidarité internationale, Sainte-Foy, Presses de l'Université du Québec, 252 p.

FAVREAU, L., et L. FRÉCHETTE (2003a). Organisation communautaire et intervention de quartier en Outaouais : l'expérience du quartier Jean-Dallaire de Hull, Cahier du Centre d'étude et de recherche en intervention sociale (CÉRIS), série Recherches, $n^{\circ} 22$, Université du Québec en Outaouais, $43 \mathrm{p}$.

FAVREAU, L., et L. FRÉCHETTE (2003b). Le développement social des communautés : le défi des nouvelles générations de travailleurs sociaux, Cahier du Centre d'étude et de recherche en intervention sociale (CÉRIS), série Recherches, $n^{\circ} 23$, Université du Québec en Outaouais, $35 \mathrm{p}$.

FAVReAu, L., et Y. HuRTUBiSe (1993). Les CLSC et les communautés locales, la contribution de l'organisation communautaire, Sainte-Foy, Presses de l'Université du Québec.

Fournier, D., J.-F. René, M. Duval, S. Garon, A. Fontaine, J. ChÉnARd et C. Lefebvre (2001). "La dynamique partenariale sur les organismes communautaires dans le contexte de la réorganisation du réseau de la santé et des services sociaux », Nouvelles pratiques sociales, vol. 14, n 1, Sainte-Foy, Presses de l'Université du Québec, p. 111-131.

FRÉCHETTE, L. (2001). «La prévention et la promotion en santé mentale : des incontournables en psychologie communautaire », dans F. Dufort (dir.) (avec la coll. de J. Guay), Agir au cœur des communautés, Québec, Les Presses de l'Université Laval, p. 217-248.

INSTITUT NATIONAL DE SANTÉ PUBLIQUE (2002a). La santé des communautés : perspectives pour la contribution de la santé publique au développement social et au développement des communautés, Québec, INSPQ, 46 p. 
INSTITUT NATIONAL DE SANTÉ PUBLIQUE (2002b). La santé des communautés : perspectives pour la contribution de la santé publique au développement social et au développement des communautés. Revue de littérature, Québec, INSPQ, 50 p.

INSTITUT NATIONAL DE SANTÉ PUBLIQUE (2002c). La santé des communautés : perspectives pour la contribution de la santé publique au développement social et au développement des communautés. Conceptions, actions, enjeux, défis et préoccupations : points de vue d'acteurs de directions de santé publique, Québec, INSPQ, 40 p.

INSTITUT NATIONAL DE SANTÉ PUBLIQUE (2002d). La santé des communautés : perspectives pour la contribution de la santé publique au développement social et au développement des communautés. Conceptions, actions, enjeux, défis et préoccupations (Document résumé), Québec, INSPQ, 4 p.

LACHAPELLE, R. (2001). CLSC et développement des communautés aujourd'hui, Cahier de la Chaire de recherche en développement communautaire (CRDC), UQAH, 24 p.

LACHAPELLE, R. (dir.) (2003). L'organisation communautaire en CLSC. Cadre de référence et pratiques, Regroupement québécois des intervenants et intervenantes en action communautaire en CLSC et centres de santé, Québec, Les Presses de l'Université Laval, 293 p.

LACHAPELLE, R. (2004). Le développement des communautés : enjeux et opportunités. Communication à la Journée d'étude intersectorielle de la Haute-Yamaska, Granby, 15 janvier 2004, 19 p.

MINISTÈRE DE LA SANTÉ ET DES SERVICES SOCIAUX (1992). La politique de la santé et du bienêtre, Québec, MSSS, $191 \mathrm{p}$.

Ministère DE LA SANTÉ ET DES SERVICES SOCIAUX (1997). Priorités nationales de santé publique 1997-2002, Québec, MSSS, 103 p.

MINISTÈRE DE LA SANTÉ ET DES SERVICES SOCIAUX (2002). Le programme de soutien aux jeunes parents. Document initial pour la première phase de l'implantation, Québec, MSSS.

Ministère de LA SANTÉ ET DES SERVICES SOCIAUX (2003). Programme national de santé publique 2003-2012, Québec, Direction générale de la santé publique, MSSS, 133 p.

NINACS, W.A. (1995). «Empowerment et service social : approches et enjeux », Service social, vol. $44, \mathrm{n}^{\circ} 1$, p. 69-93.

Ottawa (1986). Ottawa Charter for Health Promotion, First International Conference on Health Promotion. Rencontre à Ottawa, novembre 1986.

Parazelli, M., J. Hébert, F. Huot, M. Bourgon, C. Gélinas, C. Laurin, S. Lévesque, M. RHÉAUME et S. GAGNON (2003). «Les programmes de prévention précoce. Fondements théoriques et pièges démocratiques », Service social, vol. 50, p. 81-121.

PRoulX, J. (1997). Le partenariat entre l'État et les organismes communautaires dans le cadre de la loi 120 : l'enjeu de la complémentarité. Mémoire de maîtrise, Québec, Université Laval, $202 \mathrm{p}$. 
QUÉBEC (2000). Les solutions émergentes, Commission d'étude sur les services de santé et les services sociaux, Québec, Gouvernement du Québec, 408 p.

QUÉBEC (2001). Loi sur la santé publique, Québec, chapitre 60.

ThiBAULt, A., M. LEQUin et M. TREMBLAY (2000). Cadre de référence de la participation publique (démocratique, utile et crédible), Québec, Conseil de la santé et du bien-être, 23 p.

WHITE, Deena (1994). «La gestion communautaire de l'exclusion », LSP-RIAC [Revue internationale d'action communautaire], $n^{\circ} 32$, p. 37-51. 